\section{C3 Deposition in Renal Arterioles in the Loin Pain and Haematuria Syndrome}

Previous studies from this department have presented findings in a group of patients with intermittent loin pain and haematuria.$^{1}$ Renal angiograms showed abnormalities of intrarenal vessels though no changes were seen in blood vessels on biopsy of renal tissue by light microscopy. None of the patients were hypertensive or had renal tract infection or stones. The present study of fluorescent antibody staining of renal tissue and serum complement was undertaken to examine further the possible pathogenic mechanisms of the loin pain and haematuria syndrome.

\section{Methods and Results}

Fourteen specimens of renal tissue were examined. Direct staining for IgA IgG, IgM, C3, fibrin/fibrinogen (Behringwerke), C1q (Mercia Diagnostics), and albumin (Dynatech) and indirect staining for $\mathrm{C} 4, \mathrm{C} 5$, and factor $\mathrm{B}$ (Behringwerke) were undertaken. Seventy specimens of renal tissue from patients with a wide range of renal disease were also examined. A coded system of labelling was used so that the observer did not know the identity of the patient whose section was being examined. Levels of serum C1q, C3, and $\mathrm{C} 4$ were measured by radial immunodiffusion using monospecific antisera (Behringwerke). In-vivo and in-vitro C3 conversion was investigated by crossed immunoelectrophoresis using EDTA plasma and incubation of normal human serum and test serum for 45 minutes at $37^{\circ} \mathrm{C}$ respectively. ${ }^{2}$

Positive staining for $\mathrm{C} 3$ was seen in the walls of arterioles to a variable extent in all renal tissue from patients with loin pain and haematuria (see fig.). In seven cases small amounts of C3 were also seen deposited in a fine granular pattern on the glomerular basement membrane. In eight cases small amounts of fibrin/fibrinogen were also seen in glomeruli but not in blood vessels. IgA was seen on an occasional glomerular capillary loop in three cases. Staining for other complement components, IgG, IgM, and albumin was negative in all biopsies. Mesangial deposition of IgA was not seen in any case. C3 fluorescence was not abolished by washing the sections in $2 \mathrm{M}$ saline before staining. Fluoresceinated antiserum to $\mathrm{C} 3$ from another source (Mercia Diagnostics) gave identical results. Levels of Clq, $\mathrm{C} 3$, and C4 were within normal limits in all cases. No evidence of in-vivo or in-vitro C3 conversion was observed. Positive staining for C3 in renal arterioles was seen in eight other cases. Four had generalized vasculitis, two had accelerated hypertension, and in two cases the biopsy was within two weeks of delivery after pre-eclampsia.

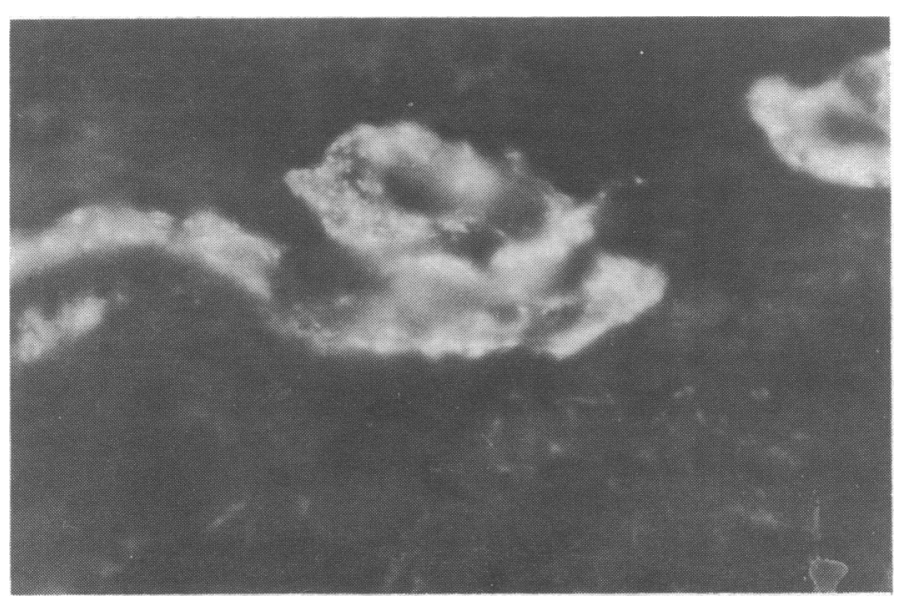

Renal biopsy section stained with fluoresceinated antihuman C3. Bright staining in wall of an arteriole.

\section{Discussion}

Staining for C3 described in this paper has not been reported before. The finding is unlikcly to be artefactual since staining could not be abolished by $2 \mathrm{M}$ saline, identical results were obtained with antiserum from two sources, and staining for proteins other than $\mathrm{C} 3$, particularly albumin, was negative. C3 in arteriolar walls in the cases of vasculitis, accelerated hypertension, and pre-eclampsia were associated with abnormalities visible on light microscopy. (Immune complex deposition might have caused $\mathrm{C} 3$ fixation in the cases of vasculitis.) No such changes were seen in the cases of loin pain and haematuria. The absence of staining for $\operatorname{IgA}$ in the glomerular mesangium clearly distinguishes our patients from those first described by Berger. ${ }^{3}$

The pathogenesis of the loin pain and haematuria syndrome remains obscure and our failure to detect immunoglobulins in blood vessel walls is unexplained. Nevertheless, complement activation without the participation of immunoglobulin is well recognized. ${ }^{4}$ Whatever the cause of the $\mathrm{C} 3$ deposition the appearances serve to characterize further the syndrome of loin pain and haematuria and support our proposal that it represents a distinct clinical and pathological entity.

We thank Miss Joan Lancaster for her skilled technical assistance and the Birmingham Regional Health Authority for a research grant

Requests for reprints should be sent to Dr. P. F. Naish.

${ }^{1}$ Burden, R. P., et al., Quarterly fournal of Medicine. In press.

2 Peters, D. K., et al., Clinical and Experimental Immunology, 1972, 11, 311

${ }^{3}$ Berger, J., Transplantation Proceedings, 1969, 1, 939.

4 Taubman, S. B., and Lepoco, I. H., Immunochemistry, 1971, 8, 951.

Department of Nephrology, North Stafis Medical Centre, Stoke-onTrent

P. F. NAISH, M.B., M.R.C.P., Consultant Physician

G. M. ABER, PH.D., F.R.C.P., Consultant Physician

W. N. BOYD, F.R.C.S., D.M.R.D., Consultant Radiologist

\section{Hypersensitivity to Chlorocresol- preserved Heparin}

Allergic reactions to heparin are rare. We have recently observed two cases of generalized and seven of local reaction to mucous heparin (Weddel Pharmaceuticals Ltd.) containing $0 \cdot 15 \%$ of chlorocresol as preservative. In all except one of these cases hypersensitivity to chlorocresol was implicated.

\section{Case Reports}

Case 1-A 35-year old woman with deep vein thrombosis and a pulmonary embolus was started on intravenous heparin. Immediately after her second dose of 10000 units of chlorocresol-preserved mucous heparin she collapsed with pallor, sweating, hypotension, and tachycardia. She recovered spontaneously after 30 minutes and was subsequently anticoagulated with warfarin alone. Intradermal skin tests gave positive reactions to both cholorcresol-preserved and preservative-free heparin.

Case 2-A 55-year-old man with deep vein thrombosis and pulmonary embolus was given 10000 units chlorocresol-preserved heparin intravenously. Over the next hour he developed nasal congestion, profuse sweating, and a generalized urticarial rash that faded within a few hours. Intradermal skin testing with preservative-free heparin (Weddel) produced no reaction and treatment was continued uneventfully using this preparation.

Cases 3-9-All these patients with proved or suspected myocardial infarction receiving prophylactic subcutaneous heparin 10000 units twice daily (given as $0.4 \mathrm{ml}$ of $25 \mathrm{MU}$ chlorocresol-preserved heparin/1). Within a few hours of the first and subsequent injections an indurated erythematous reaction developed at the site of injection. Intradermal skin testing with preservative-free heparin in four of these patients produced no response.

\section{Comment}

Adverse reactions to heparin include haemorrhage, thombocytopenia, osteoporosis, transient alopecia, and hypersensitivity phenomena. Hypersensitivity reactions are uncommon but fatal anaphylaxis has been observed after a single intravenous dose of calcium heparin. ${ }^{2}$ Such responses have been considered to be due to impurities and are nowadays extremely rare. The possibility of a contaminant in the batches of heparin used in our patients was discounted after discussion with the manufacturers. There have been other reports of Arthus-like local reactions to subcutaneous heparin, ${ }^{34}$ and one of these patients subsequently suffered anaphylaxis after intravenous heparin. ${ }^{3}$

Superficial (subdermal) injection of chlorocresol-preserved heparin may give a delayed irritant local reaction; the chlorocresol being 
avidly protein bound persists in the tissues. We could not implicate the subcutaneous injection techniques in the patients (Cases 3-9) who developed indurated pruritic transient lesions within hours of injection. Contact sensitization to cresols is recognized, ${ }^{5}$ and we suspect that all local and one of the systemic reactions seen in our patients resulted from hypersensitivity to the chlorocresol preservative. Intradermal skin testing with chlorocresol-preserved heparin gave immediate responses, whereas in only one (Case 1 ) of the tests with preservativefree heparin (Weddel) was there a reaction.

We suggest that in cases of apparent heparin sensitivity treatment may be able to continue without adverse reaction after cautious challenge with chlorocresol-free heparin.

We are grateful to Professor J. Richmond for helpful criticism, and to Mrs. E. C. Baxter (Weddel Pharmaceuticals Ltd.) for useful information.

${ }^{1}$ Moser, R. H., Clinical Pharmacology and Therapeutics, 1968, 9, 255.

2 Richmond, J., personal communication, 1975.

3 O'Toole, R. D., Annals of Internal Medicine, 1973, 79, 759.

${ }^{4}$ Hume, M., Smith-Petersen, M., and Fremont-Smith, P., Lancet, 1974, 1, 261.

5 Fisher, A. A., Contact Dermatitis, 2nd edn., p. 374. Philadelphia, Lea and Febiger, 1972.

Academic Division of Medicine, Royal Hospital, Sheffield S1 3SR B. W. HANCOCK, M.B., M.R.C.P., Lecturer ANNE NAYSMITH, M.B., B.SC., Senior House Officer

\section{Deglutition Syncope}

Syncope provoked by swallowing is rare according to the few cases reported. Probably this condition is not recognized and passes unnoticed. A salient feature of the reported cases is that in most of them disorders of the oesophagus could be shown. These ranged from diverticula ${ }^{1}$ to strictures, ${ }^{2}$ spasm, and carcinoma. ${ }^{3}$ In most cases syncope occurred mainly as a result of atrioventricular heart block, but sinus bradycardia and sinus arrest have also been reported, ${ }^{3}$ and in one case ${ }^{1}$ both sinoatrial and atrioventricular block as well as signs of other disturbances of the sinus node were recorded.

We report a case of what we suggest be termed deglutition syncope characterized by repetitive syncopes during swallowing due to heart block.

\section{Case Report}

A 43-year-old man with a past history of rheumatic fever during childhood had for several years suffered from suddenly occurring retrosternal pain, which occurred mainly during meals but also spontaneously. On many occasions he had fainted, but usually the pain was accompanied only with dizziness. Drinking of carbonated beverages regularly provoked such attacks, but no other food could be pointed out as of particular significance. The general examination, including $x$-ray examination and endoscopy of the oesophagus, was uneventful except for a left anterior hemiblock in the electrocardiogram (E.C.G.). He was monitored in the ward, and on a couple of occasions a marked variation of the heart rate was observed. Over a short period of time the heart rate could vary from 44 to 80 beats/min, showing chaotic atrial activity. A provocation test letting the patient drink carbonated water resulted in a conspicuous sinus arrythmia and bradycardia (see fig.).

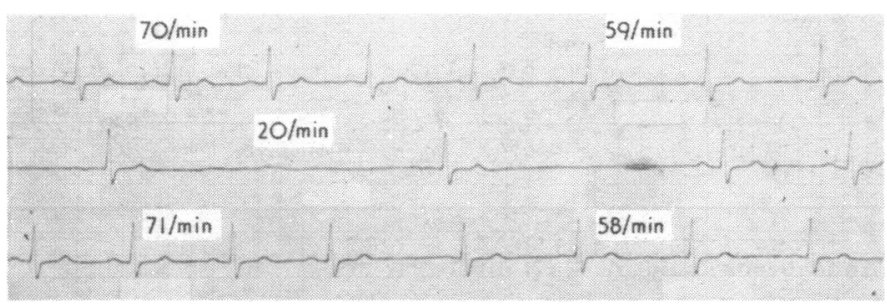

E.C.G. telemetry while swallowing, showing changing P-wave configuration and frequency slowing from 70 to 20 beats/min.
E.C.G. registration with an oesophageal lead produced atrioventricular blockade. Because of these defects of the conduction system of the heart we decided to implant an on-demand pacemaker. A control later on with inhibition of the implanted pacemaker and with the patient simultaneously drinking carbonated water showed the occurrence of an asystole lasting $3 \cdot 7$ seconds.

\section{Comment}

Local changes of the oesophagus have been considered the cause of an enhanced sensitivity of the nervous receptors so that a vagovagal reflex might be put at work with resultant heart block. Such disorders, however, are not always demonstrable, as in this report, and they alone are hardly responsible for deglutition syncope or similar conditions. The prime factor in this condition is probably a disease of the conduction system of the heart. This is supposed to exist at a subclinical level, but can be triggered by nervous reflexes originating in the oesophagus. This also applied to our patient, who according to current views suffered from the sick-sinus syndrome but who in addition displayed an atrioventricular blockade during stimulation of the nervous receptors of the oesophagus.

Deglutition can also provoke tachyarrhythmias, ${ }^{24}$ and in one reported case $^{2}$ swallowing initially produced a tachycardia of 140 beats $/ \mathrm{min}$. This suddenly changed into a sinus bradycardia of 45 beats $/ \mathrm{min}$ and syncope, but asystole did not ensue. By definition a sick sinus produces both tachyarrhythmias and bradyarrhythmias.

It is important to recognize deglutition syncope since such syncopes may be life-threatening and easily can be prevented by insertion of a pacemaker.

:James, A. H., Lancet, 1958, 1, 771.

2 Tolman, K. G., and Ashworth, W. D., Digestive Diseases, 1971, 16, 1026. 3 Tomlinson, I. W., and Fox, K. M., British Medical fournal, 1975, 2, 315.

'Bajaj, S. C., et al., Gastroenterology, 1972, 62, 632.

Medical Department, St. Josephs Hospital, 3900 Porsgrunn, Norway B. WIK, M.D., Physician

L. HILLESTAD, M.D., Physician

\section{Septic Abortion Associated with a Lippes Loop}

Septic abortion associated with an intrauterine contraceptive device (I.U.D.) has been well documented in the United States. ${ }^{1}$ The Dalkon Shield has been mainly concerned with this complication ${ }^{2} 3$ and the Food and Drug Administration suspended sales of it in the U.S.A. in June 1974. Subsequently the F.D.A. has reported 219 septic abortions and 13 deaths in association with this device. These serious complications have not been confined exclusively to the Dalkon Shield, however, and this paper reports a case of septic abortion with a Lippes Loop.

\section{Case Report}

A 26-year-old woman at 21 weeks gestation in her third pregnancy was admitted with a history of pyrexia and rigors for 24 hours and abdominal pain and vaginal bleeding of increasing severity for eight hours. She had had a Lippes Loop I.U.D. inserted two years earlier, six months after a normal pregnancy. Her first pregnancy had been terminated at 10 weeks. The current pregnancy had been uneventful up to the time of admission and she denied interference.

On admission she was severely distressed and pale. Her temperature was $38.5^{\circ} \mathrm{C}$; the blood pressure was $110 / 70 \mathrm{~mm} \mathrm{Hg}$ and the pulse rate 130 beats/ min. There was moderate lower abdominal tenderness and the uterus, the size of which was compatible with the stated period of gestation, was contracting strongly every five minutes. Vaginal bleeding was moderate; membranes bulged through the cervix, which was $2-\mathrm{cm}$ dilated. The haemoglobin was $9.6 \mathrm{~g} / \mathrm{dl}$ and the total leucocyte count $12.3 \times 10^{9} / 1$. Blood cultures subsequently grew Proteus spp. She was transfused two pints $(1 \cdot 1$ l) of blood. Ampicillin treatment, started before admission, was continued by intramuscular injection. She spontaneously aborted two hours after admission. 